\title{
VARIACIONES ENTRE ISOBIOCLIMAS (1951-1980 y 1981-2010) EN LA ESPAÑA PENINSULAR
}

\author{
María Luisa LÓPEZ FERNÁNDEZ ${ }^{1}$, Dhais PEÑA ANGULO ${ }^{2,3}$, \\ Ricardo MARCO GARCÍA ${ }^{1}$, María Soledad LÓPEZ FERNÁNDEZ ${ }^{4}$, \\ José Carlos GÓNZALEZ-HIDALGO²,3 \\ ${ }^{1}$ Departm. de Biología Ambiental, Facult. de Ciencias, Univ. de Navarra, España. \\ ${ }^{2}$ Instituto Universitario de Investigaciones en Ciencias Ambientales de Aragón, IUCA, Universidad \\ de Zaragoza, España. \\ ${ }^{3}$ Departm. de Geografia y Ordenación del Territorio, Univ. de Zaragoza, España. \\ ${ }^{4}$ Instituto de Estudios Manchegos, Ciudad Real, España. \\ mllopez@unav.es, dpang@unizar.es, rmarco@unav.es, solpfernandez@gmail.com,jicgh@unizar.es
}

\section{RESUMEN}

En el presente estudio se analizan las variaciones espaciales y temporales entre Isobioclimas de la España peninsular en dos periodos climáticos normales consecutivos (1951-1980 y 1981-2010). Los criterios aplicados para caracterizar los Isobioclimas son los de la Worldwide Bioclimatic Classification System (WBCS). El estudio se llevo a cabo con las bases de datos de alta resolución espacial de temperatura y precipitación mensual, MOTEDAS y MOPREDAS respectivamente, en su versión de malla de $10 \times 10 \mathrm{~km}$. En el primer periodo normal se han encontrado 40 Isobioclimas y 36 en el segundo. Entre ambos periodos el 38,56\% del territorio peninsular español cambia de Isobioclima, mientras que el $61,44 \%$ restante permanece estable. Considerando como movilidad de un Isobioclima su cambio de ubicación en el espacio geográfico, medible por la superficie que cede a otros, más la superficie que recibe de otros, todos los Isobioclimas se mueven y el total de los traslados asciende al 77,12\% del territorio.

Palabras clave: Bioclimatología, España, Fitotrones naturales, Variabilidad espacial, Movilidad translacional.

\begin{abstract}
In the present study spatial and temporal variations amongst Isobioclimas of mainland Spain are analyzed for two consecutive normal climatic periods (1951-1980 y 1981-2010). The criteria used for characterizing the Isobioclimates are those of the Worldwide Bioclimatic Classification System, WBCS. The study was carried out with high spatial resolution databases of monthly average temperatura and monthly rainfall, MOTEDAS and MOPREDAS, in its version of mesh 10x10 km. 40 Isobioclimas have been found in the first normal period, and 36 in the second. Between the two periods $38.56 \%$ of the Spanish mainland changes Isobioclima, and the other $61.44 \%$ remains stable. Considering as mobility of a Isobioclimaits the changes of the geographical site, measurable by the surface it yields to others, plus the surface it receives from others, all Isobioclimates change places and total transfers rises to 77.12 of the territory.
\end{abstract}


Key words: Climatology, Spain, natural growth chambers, spatial variability, translational mobility.

\section{INTRODUCCIÓN}

El análisis bioclimático es una herramienta valiosa que utiliza datos del clima para analizar el potencial ecológico de un territorio. En general, los estudios bioclimáticos realizados con trabajo de campo se refieren a zonas de escasa extensión y periodos de tiempo no muy prolongados (véase por ejemplo Gavilán et al., 1998; Ceballos et al., 2004). Por ello, las investigaciones de ámbito más general se han realizado preferentemente a partir de indicadores indirectos, como los criterios bioclimáticos (Rivas-Martínez et al., 2002 a y b), como por ejemplo las áreas de distribución potencial de especies o formaciones concretas (del Río y Penas 2006 a y b; Gavilán et al., 2007), o las relaciones generales entre clima y vegetación (Blasi et al., 1999; Bazan et al., 2015).

Una de las clasificaciones bioclimáticas más reconocidas es la denominada "Worldwide Bioclimatic Classification System", WBCS (Rivas-Martínez, 2004, 2008; Rivas-Martínez et al., 1999, 2011), desarrollada a partir de la respuesta de las comunidades vegetales a las condiciones del clima, y que concuerda perfectamente con el enfoque geosinfitosociológico de los estudios de vegetación. Se trata de un sistema jerárquico que comprende las siguientes categorías: Macrobioclima, Variante Bioclimática, Bioclima, Termotipo y Ombrotipo, cada una de ellas definida por determinados intervalos de Parámetros climáticos e Índices Bioclimáticos. La aportación fundamental de la clasificación ha sido seleccionar y, en su caso, crear, unos parámetros climáticos y unos índices bioclimáticos que captan la distribución de los geosintaxones existente en la Naturaleza. Así, la clasificación bioclimática WBCS expresa relaciones muy ajustadas entre clima, bioclima y vegetación potencial, lo que permite realizar valoraciones ecológicas de gran interés, y entre ellas la evaluación de los cambios de las condiciones del clima y su reflejo en cambios de los condicionantes de la Biodiversidad. Cada conjunto jerarquizado de las unidades bioclimáticas representa un espacio ambiental bioclimáticamente homogéneo capaz de albergar, en posición climácica, una serie de vegetación y su fauna asociada. Por estas razones, cada Isobioclima, en el que reinan unas condiciones precisas y conocidas de temperaturas y pluviosidades, incluyendo sus ritmos anuales, puede ser considerado como un fitotrón natural, cuyos límites son los umbrales superior e inferior que definen cada una de sus unidades jerárquicas (López et al., 2009). La evaluación bioclimática siguiendo los criterios de la WBCS cuenta con numerosos precedentes en el entorno Mediterráneo, no solo en España, donde aparte de los ya mencionados de Rivas-Martínez et al. (2002 a y b) se han realizado estudios globales y regionales (López Fernández et al., 2003, 2008, 2009, 2015; Piñas et al., 2008 a y b, 2009), sino también en Portugal (Mesquita y Sousa, 2009), Cerdeña (Canu et al. (2015) o Italia (Pesaresi et al., 2014).

En este trabajo se presenta el análisis de la evolución de las condiciones bioclimáticas en la España peninsular mediante el estudio de la variación en el tiempo y en el espacio de los Isobioclimas entre dos periodos normales (1951-1990 y 1981-2010). El estudio se realiza a una elevada resolución espacial, empleando las bases de datos de precipitaciones y temperaturas medias mensuales denominadas MOPREDAS y 
MOTEDAS (González-Hidalgo et al., 2011, 2015). Los objetivos principales son conocer los eventuales cambios entre Isobioclimas producidos en las décadas recientes, así como su localización espacial.

\section{MÉTODOS}

\section{1. Área de estudio}

La Península Ibérica se reconoce como un espacio de elevada variabilidad climática, que se resume en la existencia de gradientes latitudinales y longitudinales que afectan a la distribución de temperaturas, su variabilidad espacial y sus tendencias (Martín-Vide y Gil-Olcina, 2011; Peña-Angulo et al., 2015, 2016; González-Hidalgo et al., 2015), en los numerosos regímenes pluviales existentes, y en la diversidad de las tendencias de la precipitaciones mensuales, estacionales y anuales (de Luis et al., 2010; González-Hidalgo et al., 2011). La posición latitudinal, las diferencias latitudinales y longitudinal, su complejidad orográfica y la posición entre dos masas de agua contrastadas, son los principales factores que explican esta diversidad.

\section{2. "Worldwide Bioclimatic Classification System", WBCS}

La WBCS utiliza un conjunto de Parámetros climáticos y de Índices Bioclimáticos, basados en datos climáticos mensuales de temperaturas medias y de precipitaciones, correspondientes a un periodo de años estadísticamente fiable. Con los valores de estos Parámetros e Índices se pueden conocer e identificar las unidades bioclimáticas denominadas Macrobioclima, Variante Bioclimática, Bioclima, Termotipo y Ombrotipo (véase Rivas-Martínez et al., 2011). En la Tabla 1 se muestran las claves generales de la clasificación.

\begin{tabular}{|c|l|c|l|}
\hline \multicolumn{2}{|c|}{ BIOCLIMAS } & \multicolumn{2}{c|}{ TERMOTIPOS } \\
\hline Mepo & Mediterráneo pluviestacional oceánico & Tme & Termomediterráneo \\
\hline Mepc & Mediterráneo pluviestacional continental & Mte & Mesomediterráneo \\
\hline Mexo & Mediterráneo xérico oceánico & Ste & Supramediterráneo \\
\hline Teho & Templado Hiperoceánico & Tte & Termotemplado \\
\hline Teoc & Templado Oceánico & Mte & Mesotemplado \\
\hline Texe & Templado Xérico & Ste & Supratemplado \\
\hline \multicolumn{2}{|c|}{ VARIANTES } & & OMBROTIPOS \\
\hline Nrm & Normal & Sar & Semiárido \\
\hline Stp & Esteparia & Dry & Seco \\
\hline Sbm & Submediterránea & Shu & Subhúmedo \\
\hline \multicolumn{2}{|c|}{} & Hum & Húmedo \\
\cline { 2 - 4 } & Hhu & Hiperhúmedo \\
\hline
\end{tabular}

Tabla 1: Claves generales de la clasificación.

Los Isobioclimas identifican unas condiciones ambientales homogéneas y por ello son verdaderos Fitotrones naturales, ya que en su territorio la temperatura, la hu- 
medad, y el fotoperiodo están controlados naturalmente. Los Isobioclimas expresan la unidad bioclimática más sintética, compleja y, al mismo tiempo, más cercana a la realidad ambiental (López et al., 2009), y se nombran indicando todos sus componentes. Como el nombre de los Bioclimas incluye siempre el de su Macrobioclima, no hace falta repetir esta palabra. Ejemplo de nombres de Isobioclimas: Mepo Nrm Mme Dry (Mediterráneo Pluviestacional Oceánico, Normal, Mesomediterráneo, Seco), Mepo Stp Sme Shu (Mediterráneo Pluviestacional Oceánico, Estepario, Supramediterráneo Subhúmedo). El significado de las abreviaturas viene explicado en la Tabla 1.

\subsection{Datos climáticos y análisis de la variación de Isobioclimas}

Las fuentes climáticas utilizadas para la caracterización bioclimática han sido las mallas de alta resolución $\left(0,1^{\circ} \times 0,1^{\circ}\right)$, de temperaturas promedio mensuales de máximas y mínimas, y de precipitaciones mensuales (periodo 1951-2010), procedentes de las bases de datos denominadas MOPREDAS y MOTEDAS elaboradas a partir de un control de calidad y reconstrucción de las fuentes documentales almacenadas en los archivos de AEMet (González-Hidalgo et al., 2011, 2015). Con estos datos climáticos y los criterios de la WBCS, se identificaron los Isobioclima de cada celda de la malla en los dos periodos normales analizados (1951-1980 y 1981-2010). El contraste de ambos periodos permitió identificar en cada Isobioclima su movilidad y estabilidad. La movilidad estima el cambio en el espacio de un Isobioclima; no es un concepto areal, porque cada celda de la malla que cambia de Isobioclima cuenta dos veces, una porque el Isobioclima de partida pierde representación espacial al desaparecer, y otra porque el Isobioclima de llegada cambia su distribución al recibirlo. La estabilidad de un Isobioclima se contabiliza calculando el área que conserva la misma calificación isobioclimática de un periodo al otro, es por tanto un concepto areal.

\section{RESULTADOS}

\subsection{Presencia, distribución y cambio de Isobioclimas (1951-1980 y 1981-2010)}

En la tabla 2 se muestran, numerados del 1 al 44, los Isobioclimas identificados en los dos periodos, cuya distribución se ofrece en la figura 1.

El total de Isobioclimas en el periodo 1951-1980 es de 40, frente a 36 en el segundo, ocupando los 18 Mediterráneos 4/5 del territorio, y los 26 Templados el 1/5 restante. Esta diversidad no debe ocultar que solamente tres Isobioclimas ocupaban el $60 \%$ y $55 \%$ del territorio en los dos periodos. Los tres Isobioclimas con más extensión territorial, en el primer periodo, son: 3 Mepo Nrm Mme Dry, 7 Mepo Nrm Sme Shu y 4 Mepo Nrm Mme Shu; y en el $2^{\circ}$ periodo, 3 Mepo Nrm Mme Dry, 7 Mepo Nrm Sme Shu y 1 Mepo Nrm Tme Dry. Son todos ellos mediterráneos y ocupan: el 1, la Submeseta Sur, Extremadura, y gran parte de Andalucía, y de la costa Este, y del Valle del Ebro; el 7, gran parte de la Submeseta Norte y Sistema Ibérico; el 4, gran parte de los sistemas montañosos de la mitad Sur peninsular, y algo en la vertiente Sur del Pirineo; y el 1, el Valle del Guadalquivir y las costas Sur y Este peninsulares.

En el cambio de periodo, 8 de los Isobioclimas presentes desaparecen, y hay 4 nuevos, inexistentes con anterioridad, si bien estos 12 Isobioclimas tienen todos una mínima representación territorial (Tabla 2). 


\begin{tabular}{|c|c|c|c|c|c|c|c|c|}
\hline $\mathbf{N}$ & Tipo & $\begin{array}{l}1951- \\
1980\end{array}$ & $\begin{array}{l}1981- \\
2010\end{array}$ & Variación & Cede & Gana & Movilidad & Estabilidad \\
\hline 1 & Mepo Nrm Tme Dry & 1,45 & 8,37 & 6,92 & 0,00 & 6,92 & 6,92 & 1,45 \\
\hline 2 & Mepo Nrm Tme Shu & 0,40 & 1,95 & 1,55 & 0,17 & 1,72 & 1,89 & 0,23 \\
\hline 3 & Mepo Nrm Mme Dry & 32,84 & 34,49 & 1,64 & 6,73 & 8,37 & 15,09 & 26,12 \\
\hline 4 & Mepo Nrm Mme Shu & 11,85 & 8,06 & $-3,78$ & 7,36 & 3,57 & 10,93 & 4,49 \\
\hline 5 & Mepo Nrm Mme Hum & 0,69 & 1,24 & 0,55 & 0,67 & 1,22 & 1,89 & 0,02 \\
\hline 6 & Mepo Nrm Sme Dry & 3,69 & 5,90 & 2,22 & 0,11 & 2,33 & 2,45 & 3,57 \\
\hline 7 & Mepo Nrm Sme Shu & 15,97 & 12,46 & $-3,52$ & 5,66 & 2,14 & 7,80 & 10,32 \\
\hline 8 & Mepo Nrm Sme Hum & 4,97 & 2,25 & $-2,71$ & 3,04 & 0,32 & 3,36 & 1,93 \\
\hline 9 & Mepo Nrm Sme Hhu & 0,02 & 0,00 & $-0,02$ & 0,02 & 0,00 & $\mathbf{0 , 0 2}$ & 0,00 \\
\hline 10 & Mepo Stp Mme Dry & 4,13 & 2,87 & $-1,26$ & 2,25 & 0,99 & 3,25 & 1,87 \\
\hline 11 & Mepo Stp Mme Shu & 0,21 & 0,15 & $-0,06$ & 0,21 & 0,15 & 0,36 & 0,00 \\
\hline 12 & Mepo Stp Sme Dry & 0,61 & 1,32 & 0,71 & 0,38 & 1,09 & 1,47 & 0,23 \\
\hline 13 & Mepo Stp Sme Shu & 0,88 & 0,38 & $-0,50$ & 0,82 & 0,32 & 1,15 & 0,06 \\
\hline 14 & Mepc Nrm Mme Dry & 0,00 & 0,34 & 0,34 & 0,00 & 0,34 & 0,34 & 0,00 \\
\hline 15 & Mepc Nrm Mme Shu & 0,13 & 0,13 & 0,00 & 0,04 & 0,04 & $\mathbf{0 , 0 8}$ & 0,10 \\
\hline 16 & Mexo Nrm Tme Sar & 0,61 & 2,62 & 2,01 & 0,00 & 2,01 & 2,01 & 0,61 \\
\hline 17 & Mexo Nrm Mme Sar & 3,11 & 1,47 & $-1,64$ & 1,97 & 0,32 & 2,29 & 1,15 \\
\hline 18 & Mexo Stp Mme Sar & 0,00 & 0,02 & 0,02 & 0,00 & 0,02 & 0,02 & 0,00 \\
\hline 19 & Teho Nrm Tte Hum & 0,21 & 0,13 & $-0,08$ & 0,11 & 0,04 & 0,15 & 0,10 \\
\hline 20 & Teho Nrm Tte Hhu & 0,00 & 0,21 & 0,21 & 0,00 & 0,21 & 0,21 & 0,00 \\
\hline 21 & Teho Nrm Mte Hum & 0,21 & 0,04 & $-0,17$ & 0,17 & 0,00 & 0,17 & 0,04 \\
\hline 22 & Teho Sbm Tte Hum & 1,05 & 1,24 & 0,19 & 0,46 & 0,65 & 1,11 & 0,59 \\
\hline 23 & Teho Sbm Tte Hhu & 0,65 & 0,13 & $-0,52$ & 0,52 & 0,00 & $\mathbf{0 , 5 2}$ & 0,13 \\
\hline 24 & Teho Sbm Mte Hum & 1,36 & 0,25 & $-1,11$ & 1,11 & 0,00 & 1,11 & 0,25 \\
\hline 25 & Teoc Nrm Tte Hum & 0,10 & 0,40 & 0,31 & 0,08 & 0,38 & 0,46 & 0,02 \\
\hline 26 & Teoc Nrm Mte Shu & 0,06 & 0,00 & $-0,06$ & 0,06 & 0,00 & 0,06 & 0,00 \\
\hline 27 & Teoc Nrm Mte Hum & 1,36 & 0,94 & $-0,42$ & 0,75 & 0,32 & 1,07 & 0,61 \\
\hline 28 & Teoc Nrm Mte Hhu & 0,25 & 0,00 & $-0,25$ & 0,25 & 0,00 & 0,25 & 0,00 \\
\hline 29 & Teoc Nrm Ste Shu & 0,02 & 0,00 & $-0,02$ & 0,02 & 0,00 & $\mathbf{0 , 0 2}$ & 0,00 \\
\hline 30 & Teoc Nrm Ste Hum & 1,83 & 1,38 & $-0,46$ & 0,53 & 0,08 & $\mathbf{0 , 6 1}$ & 1,30 \\
\hline 31 & Teoc Nrm Ste Hhu & 0,04 & 0,00 & $-0,04$ & 0,04 & 0,00 & 0,04 & 0,00 \\
\hline 32 & Teoc Stp Mte Shu & 0,50 & 0,88 & $\mathbf{0 , 3 8}$ & 0,29 & 0,67 & 0,96 & 0,21 \\
\hline 33 & Teoc Stp Mte Hum & 0,02 & 0,02 & 0,00 & 0,02 & 0,02 & 0,04 & 0,00 \\
\hline 34 & Teoc Stp Ste Shu & 0,40 & 0,13 & $-0,27$ & 0,40 & 0,13 & $\mathbf{0 , 5 3}$ & 0,00 \\
\hline 35 & Teoc Sbm Tte Shu & 0,31 & 0,13 & $-0,17$ & 0,31 & 0,13 & 0,44 & 0,00 \\
\hline 36 & Teoc Sbm Tte Hum & 0,17 & 1,53 & 1,36 & 0,00 & 1,36 & 1,36 & 0,17 \\
\hline 37 & Teoc Sbm Tte Hhu & 0,04 & 0,10 & 0,06 & 0,04 & 0,10 & $\mathbf{0 , 1 3}$ & 0,00 \\
\hline 38 & Teoc Sbm Mte Shu & 1,09 & 0,80 & $-0,29$ & 0,71 & 0,42 & 1,13 & 0,38 \\
\hline 39 & Teoc Sbm Mte Hum & 5,31 & 5,75 & 0,44 & 1,18 & 1,62 & 2,81 & 4,13 \\
\hline 40 & Teoc Sbm Mte Hhu & 0,02 & 0,00 & $-0,02$ & 0,02 & 0,00 & 0,02 & 0,00 \\
\hline 41 & Teoc Sbm Ste Shu & 0,38 & 0,00 & $-0,38$ & 0,38 & 0,00 & $\mathbf{0 , 3 8}$ & 0,00 \\
\hline 42 & Teoc Sbm Ste Hum & 2,90 & 1,87 & $-1,03$ & 1,53 & 0,50 & 2,03 & 1,38 \\
\hline 43 & Teoc Sbm Ste Hhu & 0,17 & 0,00 & $-0,17$ & 0,17 & 0,00 & 0,17 & 0,00 \\
\hline 44 & Texe Stp Mte Shu & 0,00 & 0,04 & 0,04 & 0,00 & 0,04 & 0,04 & 0,00 \\
\hline
\end{tabular}

Tabla 2: Cambios entre periodos de Isobioclimas. Datos expresados en porcentaje de superficie sobre el total. 
Por último, durante el segundo periodo 17 Isobioclimas ganan extensión geográfica, 25 Isobioclimas la pierden, y 2 mantienen sus áreas. Los 3 Isobioclimas que más territorio ganan en el $2^{\circ}$ periodo son: : 1 Mepo Nrm Tme Dry, 6 Mepo Nrm Sme Dry y 16 Mexo Nrm Tme Sar; los 3 Isobioclimas que más territorio pierden en el $2^{\circ}$ periodo son: 4 Mepo Nrm Mme Shu, 7 Mepo Nrm Sme Shu y 8 Mepo Nrm Sme Hum (Ver Tabla 2 y Figura 1).
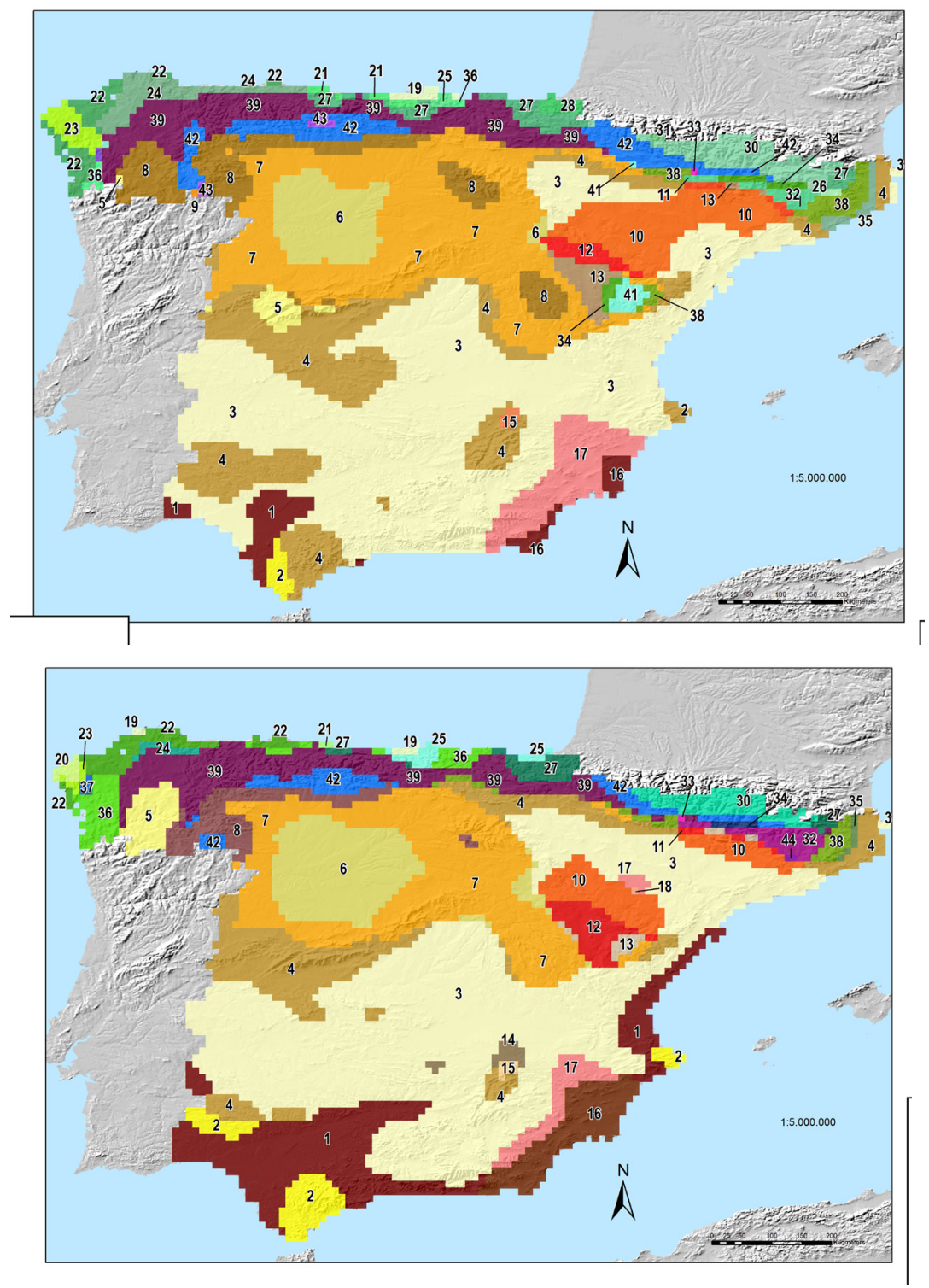

Fig. 1: Extensión y localización de los Isobioclimas en primer (arriba) y segundo (abajo) periodos analizados. Los números se corresponden con los de la Tabla 1. Elaboración propia. 


\subsection{Movilidad y estabilidad de los Isobioclimas}

En términos globales el 38,56\% del territorio, más $1 / 3$ del mismo, cambia su Isobioclima original. Es decir, la movilidad de los Isobioclimas ha afectado a una superficie equivalente al $77,12 \%$ del territorio (Tabla 2 y figura 2 , arriba). Por su parte, la estabilidad de los Isobioclimas se ha detectado en el 61,44\% del total del territorio, en donde se mantiene el mismo Isobioclima entre periodos (Tabla 2 y figura 2 , abajo). Los Isobioclimas que más ganan son: 1,6 y 16; los que más pierden: 4, 7 y 8; y los que más se mueven: $3,4,7,1$. A su vez, los mas estables son: 3, 7, 4 y 39.
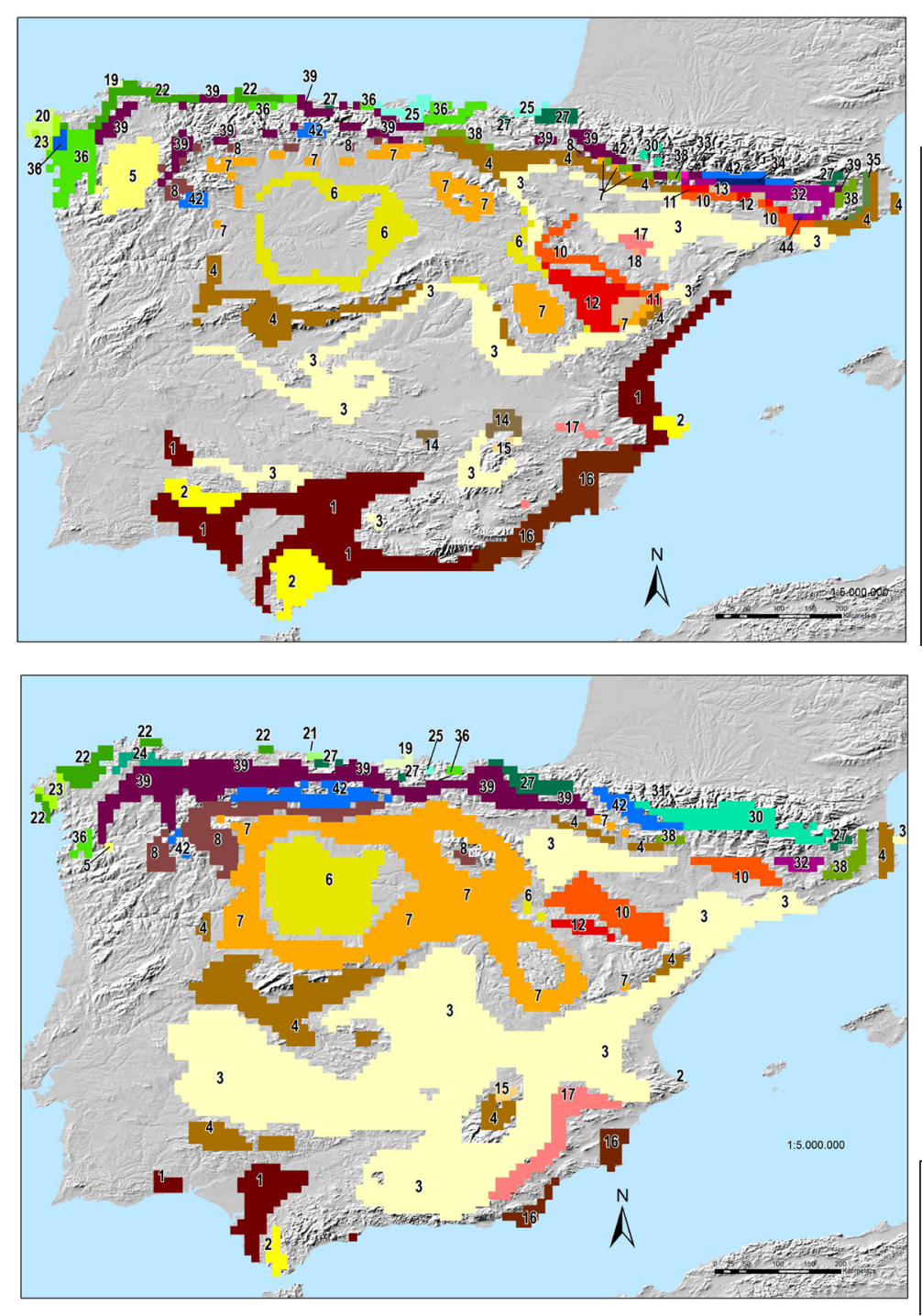

Figura 2: Movilidad (arriba) y Estabilidad (abajo) de los Isobioclimas con el cambio de treintena. Los números se corresponden con los de la Tabla 1. Elaboración propia. 


\section{DISCUSIÓN}

El análisis de las variaciones en el tiempo de los Isobioclimas en la España peninsular permite considerar dos aspectos diferentes, en primer lugar las variaciones de la superficie ocupada, y en segundo lugar el cambio de su distribución (movilidad).

\subsection{Cambio en la superficie ocupada por los Isobioclimas}

Las variaciones de la superficie ocupada por cada Isobioclima, entre las dos treintenas consideradas, es una indicación de cómo las condiciones climáticas pueden haber diferido espacialmente en el tiempo en dichas áreas. En general, los Isobioclimas que más territorio ganan en el segundo periodo, así como los que aparecen como nuevos, pertenecen a los siguientes: Bioclimas: Mepo, Mexo, Mepc y Texe; a la Variante esteparia, Stp; a los Termotipos Tme y Tte; y, finalmente, a los Ombrotipos semiárido (Sar) y seco (Dry). A su vez, los Isobioclimas que más territorio pierden, así como los que desaparecen, en el segundo periodo, pertenecen al Bioclima Teoc, a la Variante Sbm, a los Termotipos Sme y Ste; y a los Ombrotipos Shu, Hum e Hhu. Estos cambios suponen, en general, que en el segundo periodo aumenta la superficie caracterizada por los Isobioclimas con Xericidad estival, con Variante esteparia, con Termotipos cálidos y con Ombrotipos semiáridos y secos.

\subsection{Cambio en la localización de los Isobioclimas: su Movilidad y su Estabilidad}

Aunque la estabilidad isobioclimática del territorio es relativamente grande y el $61,44 \%$ del territorio mantiene su calificación isobioclimática entre periodos, el cambio detectado parece afectar intensamente a la distribución espacial de los Isobiclimas porque, tal como hemos definido la movilidad, si el 38,56\% de pixeles cambia de Isobioclima, ello supone doble \% de movilidad, ya que los Isobioclimas perdidos son reemplazados por otros. Es decir, ese cambio de calificación hay que multiplicarlo por dos al considerar la movilidad, que en nuestro caso asciende al 77,12 \% del territorio. Hay que hacer notar que el movimiento de traslación motivado por el cambio de las condiciones del clima afecta, en mayor o menor medida, a todos los Isobioclimas. También es interesante constatar que, prácticamente, los Isobioclimas más extensos, $\mathrm{n}^{\mathrm{o}} 3,7$ y 4, son, al mismo tiempo, los que más se mueven y también los más estables (por orden los $n^{\circ} 374$ ); cabe pensar que su gran extensión territorial les permite a la vez perder y ganar mucho, pero manteniendo estable gran parte de su territorio.

La movilidad se localiza, en general, en las vertientes montañosas así como en las periferias de las depresiones del Duero y Ebro y costa Mediterránea.

\subsection{Síntesis final}

El análisis de las variaciones en las condiciones bioclimáticas en España peninsular durante las décadas recientes permite identificar las variaciones de la superficie ocupada, así como la movilidad de los Isobioclimas, o fitotrones naturales. Todos los Isobioclimas -menos dos-, han variado su área de distribución, y en todos se han observado cambios de localización, lo que significa que las condiciones potenciales de las comunidades vegetales y animales asociadas a cada Isobioclima han variado a lo largo del periodo analizado. Aquellas áreas en donde se han detectado dichos 
cambios podrían quizá ser consideradas como de interés preferente para analizar las variaciones de las condiciones del clima y sus relaciones con los sistemas naturales.

\section{AGRADECIMIENTOS}

Ministerio de Ciencia e Innovación, Gobierno de España, Proyecto DESEMON, CGL2014-52135-C3-3-R. Gobierno Regional de Aragón DGA-FSE (Grupo de Investigación Consolidado "Clima, Agua, Cambio Global y Sistemas Naturales"). Dhais Peña-Angulo es becaria de doctorado del Programa FPI del Ministerio de Ciencia e Innovación. Datos climáticos originales cedidos por AEMET.

\section{REFERENCIAS}

Bazan, G., Marino, P., Guarino, R., et al. (2015). Bioclimatology and vegetation series in Sicily: a geostatistical approach. Annales Botanici Fennici, 52, pp. 1-18.

Blasi, C., Carranza, M.L., Filesi, L., et al. (1999). Relation between climate and vegetation along a Mediterranean-Temperate boundary in central Italy. Global Ecology and Biogeography, 8, pp. 17-27.

Canu, S., Rosati, L., Fiori, M., et al. (2015). Bioclimate map of Sardinia (Italy). Journal of Maps, 11, pp. 711-718.

Ceballos, A., Martínez, J., Luengo, M.A. (2004). Analysis of rainfall trends and dry periods on a pluviometric gradient representative of Mediterranean climate in the Duero Basin, Spain. Journal of Arid Environment, Volumen 58, pp. 214-232.

de Luis, M., Brunetti, M., González-Hidalgo, J.C., et al. (2010). Changes in seasonal precipitation in the Iberian Peninsula during 1946-2005. Global Planetary Changes. 74, pp. 27-33.

del Río, S. and Penas, A. (2006 a). Potential distribution of semi-deciduous forests in Castile and Leon (Spain) in relation to climatic variations. Plant Ecology, Volumen 185, pp. 269-282.

del Río, S. and Penas, A. (2006 b). Potential areas of evergreen forests in Castile and Leon (Spain) according to future climate change. Phytocoenologia, 36, pp.45-66.

Gavilán, R.G., Fernandez-Gonzalez, F., Blasi, C. (1998). Climatic classification and ordination of the Spanish Sistema Central: relationships with potential vegetation. Plant Ecology. Volumen 139, pp. 1-11.

Gavilán, R.G., Sanchez, D., Vilches, B., et ál. (2007). Modeling current distribution of Spanish Quercus pyrenaica forests using climatic parameters. Phytocoenologia, Volumen 37, pp. 561-581.

González-Hidalgo, J,C,, Brunetti, M., de Luis, M. (2011). A new tool for monthly precipitation analysis in Spain: MOPREDAS database (monthly precipitation trends Dec. 1945-Nov. 2005). Internt. Jour. of Climatology, Vol. 31, pp.715-731.

González-Hidalgo, J.C., Peña-Angulo, D., Brunetti, M., Cortesi, N. (2015). MOTEDAS: a new monthly temperature database for mainland Spain and the trend in temperature (1951-2010). Internt. Jour. of Climatology, Vol. 35, pp. 4444-4463. 
López Fernández, M. L.; Marco, R.; Piñas, S.; \& López F., M.S. (2015). Mapa Isobioclimático de la España Peninsular y Balear. Documentos Aljibe on-line, vol. II, no 4.7 de octubre de 2015. Ciudad Real. Edita Sociedad Surcos. http:// www.naturalezaenhispania.com.

López Fernández, M. L.; Piñas, S.; Amézketa Ibero, A.; Aquerreta Molina, S.; López, M.S.; Almárcegui, I.; Urdiain, M.; Royo Esnal, A. (2003). Mapa bioclimático de Navarra. Publicaciones de Biología. Universidad de Navarra, Serie Botánica. Pamplona, Volumen 15: pp 53-63.

López Fernández, M. L., Piñas, S. y López, M. S. (2008). Macrobioclimas, Bioclimas y Variantes Bioclimáticas de la España Peninsular y Balear, y su cartografía. Publ. de Biología, Univ. de Navarra Serie Botánica, Vol. 17:pp 229-236.

López, M. L., Piñas, S. y López, M.S. (2009). Isobioclimas de la provincia de Málaga y su Cartografía. En Biogeografia Scientia Biodiversitatis (Actas del V Congreso Español de Biogeografía. Málaga 9-12 de Septiembre (2008) Real, R \& Márquez, A.Ñ.. Editores. Málaga.

Martín-Vide, J. y Gil Olcina, J. (2001). Tiempos y climas de España. Madrid, Alianza Editorial. Col. El libro universitario, 264 pp.

Mesquita, S. and Sousa, A.J. (2009). Bioclimatic mapping using geostatistical approaches: application to mainland Portugal. Internatinal Journal of Climatology, 29, pp. 2156-2170.

Peña-Angulo, D., Cortesi, N., Brunetti, M., González-Hidalgo, J.C. (2015). Spatial variability of maximum and minimum monthly temperature in Spain during 1981-2010, evaluated by Correlation Decay Distance (CDD). Theoretical and Applied Climatology, Volumen 122, pp. 35-45.

Peña-Angulo, D., Brunetti, M., Cortesi, C., Gonzalez-Hidalgo, J.C. (2016). A new climatology of maximum and minimum temperature (1951-2010) in the Spanish mainland. International Journal of Geographical Information Science, (en prensa DOI 10.1080/13658816.2016.1155712).

Pesaresi, S., Galdenzi, D., Biondi, E., \& Casavecchia, S. (2014). Bioclimate of Italy: application of the worldwide bioclimatic classification system, Journal of Maps, DOI: $10.1080 / 17445647.2014 .891472$

Piñas, S., López, F., M.S. y López Fernández, M.L. (2008a). Termotipos de la España Peninsular y Balear, y su cartografía. Publicaciones de Biología de la Universidad de Navarra. Serie Botánica, Volumen 17, pp 237-242.

Piñas, S., López, F., M.S. y López Fenández, M.L. (2008b). Ombrotipos de la España Peninsular y Balear, y su cartografía. Publicaciones de Biología de la Universidad de Navarra. Serie Botánica, Volumen 17, pp 243-248.

Piñas, S., López, M.L. \& López Fernández, M.S. (2009). Andalucía: Cartografía y áreas de sus Bioclimas, Continentalidad, Termotipos y Ombrotipos. En "Biogeografía Scientia Biodiversitatis". (Actas del V Congreso Español de Biogografía. Málaga 9-12 de septiembre 2008). Ed. Real, R. y Márquez, A.L., pp. 109-119.

Rivas-Martínez, S. (2008). Globalbioclimatics, Internet: http:// www. globalbioclimatics.org.

Rivas-Martínez, S., Rivas Sáenz, S., Penas, A. (2011). Worldwide bioclimatic classification system. Global Geobotany, 634 pp +4 . 